\title{
Nuevos registros de poblaciones de Corynabutilon ochsenii (Phil.) Kearney a una mayor altitud, en formaciones vegetales post incendio en la Reserva Nacional Malleco, Chile
}

\author{
New records of Corynabutilon ochsenii (Phil.) Kearney populations at high elevation \\ post fire in the Malleco National Reserve, Chile
}

\author{
Zoia Neira ${ }^{1}$, Marcelo Saavedra², Soraya Calzadilla ${ }^{1}$, Marcelo Hernández ${ }^{1}$ \& Cecilia Obreque ${ }^{3}$ \\ 1Departamento de Ciencias Forestales y Laboratorio Silvicultura, Universidad de La Frontera, Casilla 54-D, Temuco, Chile. \\ 2Departamento Áreas Silvestres Protegidas, Región de la Araucanía, CONAF, Francisco Bilbao 931, 2do. Piso, Temuco. Chile. \\ ${ }^{3}$ Ingeniería en Recursos Naturales, Universidad de La Frontera, Casilla 54-D, Temuco, Chile.
}

\begin{abstract}
We report new records of Corynabutilon ochsenii (Phil.) Kearney populations in areas affected by forest fires 12 years ago at the Malleco National Forest Reserve in southern Chile. One of the populations is found at $1350 \mathrm{~m}$ a.s.1., which is the highest altitude ever reported for this species. $C$. ochsenii is part of the species that are reestablishing after the fire.
\end{abstract}

Corynabutilon ochsenii (Phil.) Kearney, es un arbusto endémico de Chile, conocido comúnmente como Huella chica. Es una especie extremadamente escasa (Hoffmann 1982) y actualmente está amenazada de extinción y es una de las menos conocidas del país (Hauenstein \& Saavedra 2011). Huella chica es catalogada según el Libro Rojo de la Flora Terrestre de Chile (Benoit 1989) como Rara, actualmente de acuerdo al Reglamento de Clasificación de Especies Silvestres del Ministerio de Medio Ambiente, la clasificó como Casi Amenazada (NT) (Ministerio del Medio Ambiente 2013).

$\mathrm{Su}$ rango de distribución es reducido, encontrándose registros desde la Región de la Araucanía, Provincia de Malleco (3754'S), hasta la Región de los Lagos, Provincia de Osorno (40 $14^{\circ}$ 'S), tanto en la Cordillera de la Costa como en el Valle central y la Precordillera Andina. Se le asocia creciendo mezclado con otros arbustos del matorral húmedo de la provincia de Valdivia (Hoffmann 1982) y dentro del tipo forestal Roble-Raulí-Coigüe, específicamente dentro del subtipo remanentes originales del bosque de RobleLaurel-Lingue-Coigüe (Hechenleitner et al. 2005). Es de gran valor ornamental, cultivándose tanto en el país como en el extranjero (Riedemann \& Aldunate 2003, Hechenleitner et al. 2005).

Se caracteriza por ser un arbusto ramoso, bajo, de entre 1 a $2,5 \mathrm{~m}$ de altura, con un diámetro de tronco de hasta $5 \mathrm{~cm}$, de ramas delgadas y flexibles, con follaje ralo y pubescente, con pelos simples en la cara superior (adaxial) y estrellados en la inferior (abaxial) (Donoso \& Ramírez 1985, Hoffmann 1991, Riedemann \& Aldunate 2003). Sus flores son solitarias o bifloras, con 5 pétalos violáceos, y sus frutos son cápsulas agrupadas en 6 a 10 mericarpos (Serra et al. 1986, Marticorena 2005, Hauenstein \& Saavedra 2011). Corynabutilon ochsenii pertenece a la familia Malvaceae, la que mundialmente agrupa a 243 géneros y 4.300 especies; en Chile está representada por 20 géneros y 90 especies (Marticorena 2005). Esta familia es de distribución cosmopolita, aunque prospera mejor en las regiones tropicales y subtropicales del mundo. Se caracteriza por estar constituida por arbustos y hierbas.

De la Huella chica se dispone de información muy fragmentada acerca de sus poblaciones, tanto de su ubicación como de su abundancia, y de las comunidades vegetales en que habita en la Región de la Araucanía. Se reporta en pocos lugares, falta mayor información y datos sobre el hábitat y condiciones de la especie, y en muchos de estos lugares hoy, aparentemente, ya no está presente o los registros son de data muy antigua (Hauenstein \& Saavedra 2011).

Su presencia es advertida comúnmente como ejemplares aislados, de pequeña superficie, sobre suelos de origen volcánico profundos (trumaos), desde el nivel del mar hasta los 600 m s.n.m. en la parte sur de su distribución en la vertiente oriental de la Cordillera de la Costa (Hechenleitner et al. 2005), y hasta los $900 \mathrm{~m}$ s.n.m. en la parte norte de su distribución en la Reserva Nacional Malleco, registro reportado por Saavedra (2007) como antecedente técnico para un plan de conservación de esta especie. Esta comunicación breve, aporta nuevos antecedentes y registro de seis poblaciones de Huella chica encontradas en una unidad del Sistema Nacional de Áreas Protegidas del Estado (SNASPE), creciendo en sectores con posterioridad al gran incendio forestal del año 2002, situadas desde los 835 hasta 
los 1.350 m s.n.m., considerándose esta última la población de mayor altitud hasta hoy reportada, hallazgos realizados entre los años 2012-2014 (Tabla I, Fig. 1).

Estas poblaciones se encuentran creciendo dentro del tipo forestal Roble-Raulí-Coigüe (Donoso 1981) en el sector Prado 18 (Tabla I), las que se originaron principalmente de semilla, donde un alto porcentaje de los ejemplares presenta flores y frutos. Las especies acompañantes son: Nothofagus obliqua (Mirb.) Blume, N. alpina (Poepp. et Endl.) Oerst., $N$. dombeyi (Mirb.) Blume, Persea lingue (Ruiz \& Pav.) Nees, Gevuina avellana Molina, Aristotelia chilensis (Molina) Stuntz., Lomatia dentata (Ruiz \& Pav.) R.Br., Ribes magellanicum Poir., Boquila trifoliolata (DC.) Dcne, Rubus ulmifolius Schott. En el sector de Niblinto (Tabla I) se desarrolla en el tipo forestal Lenga la población Macizo Sur Oeste, y las demás rodeadas del tipo forestal Coigüe-RaulíTepa (Donoso 1981). Las principales especies acompañantes son N. dombeyi, N. alpina, Laureliopsis philippiana (Looser) Schodde, N. pumilio (Poepp. et Endl.) Krasser, Aristotelia chilensis, Lomatia dentata, Maytenus magellanica (Lam.) Hook.f., Chusquea culeou E. Desv., Ribes magellanicum, Azara microphylla Hook.f., Gaultheria phillyreifolia (Pers.) Sleumer.

En la actualidad, los factores antrópicos imperantes como la deforestación, la fragmentación del paisaje, los cambios de uso de suelo, entre otros, tienen a esta especie en condiciones de alta vulnerabilidad, por lo que se requiere medidas inmediatas de conservación con el fin de prevenir disminuciones posteriores (Hechenleitner et al. 2005). Además, el escaso conocimiento de su estado de conservación a lo largo de su rango de distribución, hace necesario emprender acciones que aporten a su conocimiento, considerando la localización de las actuales poblaciones, las características de su hábitat y la forma de propagación (Ross 1987, Hechenleitner et al. 2005). Esta situación ha llevado a emprender por parte de CONAF el Plan Nacional de Conservación de la Huella chica Corynabutilon ochsenii en Chile, que tiene por objetivo general conservar la diversidad biológica de la huella chica, mediante el desarrollo de acciones destinadas a conservar y recuperar sus poblaciones y su hábitat, tanto en terrenos fiscales como privados, siendo una de las 13 especies elegidas para tales fines (Hauenstein \& Saavedra 2011).

Materiales estudiados: Región de La Araucanía, Malleco, Prado 18, Guardería. 835 m, 26-III-2015, Z. Neira \& M. Saavedra (CONC 179891). Niblinto, Pichimalleco, 800 m, 26-III-2015, Z. Neira \& M. Saavedra (CONC 180154). Niblinto, Las Escuadras, 900 m, 26-III-2015, Z. Neira \& M. Saavedra (CONC 180156). Niblinto, Los Mapochos, 1000 m, 2-IV-2015, Z. Neira \& M. Saavedra (CONC 180157). Niblinto, Macizo Sur Oeste, 1200 m, 9-IV-2015, Z. Neira \& M. Saavedra (CONC 180158). Niblinto, Las Quemas, 1350 m, 10-IV-2015, Z. Neira \& M. Saavedra (CONC 180155).

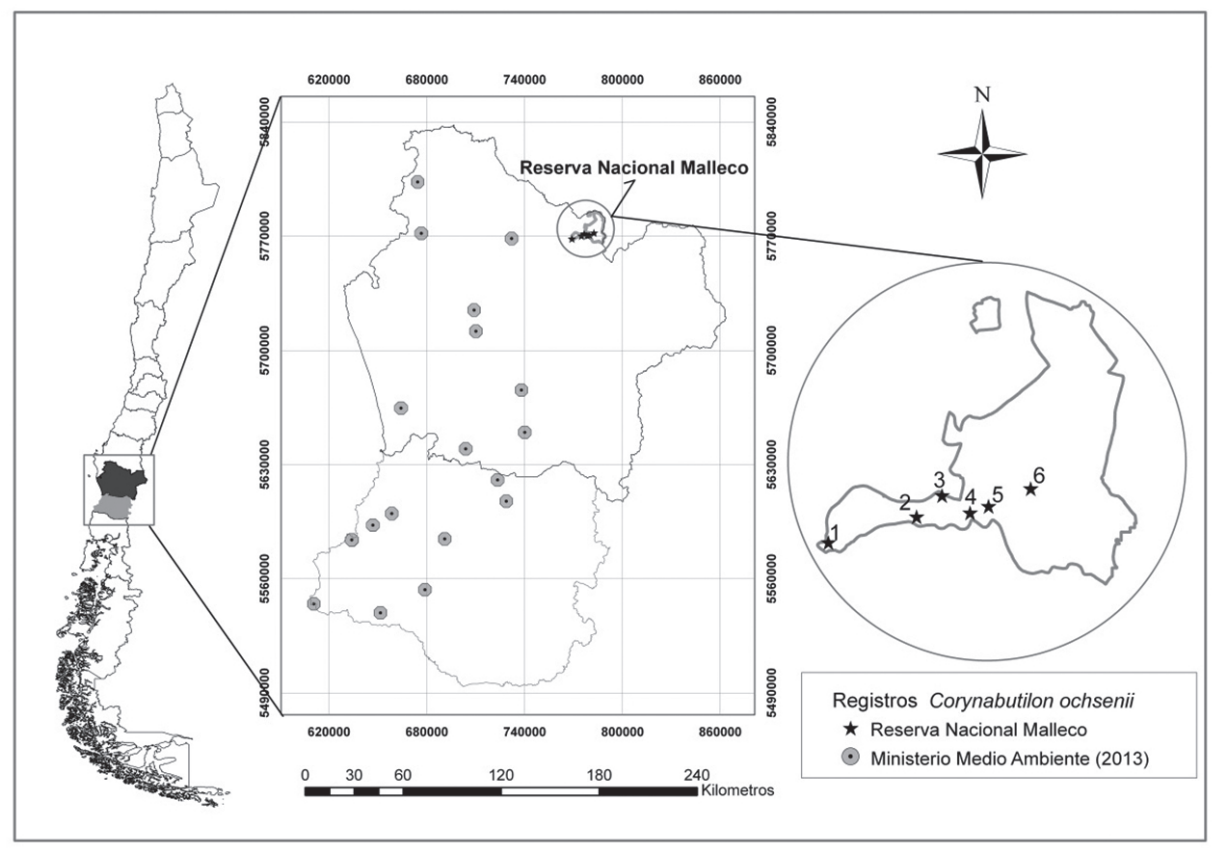

Figura 1. Ubicación de las nuevas poblaciones de C. ochsenii en la Reserva Nacional Malleco. 1. Guardería, 2. Pichimalleco, 3. Las Escuadras, 4. Los Mapochos, 5. Macizo Sur Oeste, 6. Las Quemas.

Figure 1. Locating new populations of $C$. ochsenii in Malleco National Reserve. 1. Guardería, 2. Pichimalleco, 3. Las Escuadras, 4. Los Mapochos, 5. Macizo Sur Oeste, 6. Las Quemas. 
TABLA 1. Localización y registros de nuevas poblaciones reportadas de C. ochsenii en la Reserva Nacional Malleco.

TABLE 1. Localization and records of new populations of $C$. ochsenii reported in Malleco National Reserve.

\begin{tabular}{|c|c|c|c|c|c|}
\hline \multirow[t]{2}{*}{ SeCtor } & \multirow[t]{2}{*}{ POBLACIÓN } & \multirow{2}{*}{$\begin{array}{l}\text { Altitud } \\
\text { (m s.n.m.) }\end{array}$} & \multicolumn{2}{|c|}{ COORDEnadas } & \multirow{2}{*}{$\begin{array}{l}\text { NúMERO DE } \\
\text { INDIVIDUOS }\end{array}$} \\
\hline & & & Latitud $(\mathrm{O})$ & LONGITUD (S) & \\
\hline Prado 18 & Guardería & 835 & $38^{\circ} 11^{\prime} 28,14^{\prime \prime}$ & $71^{\circ} 55^{\prime} 41,80^{\prime \prime}$ & 31 \\
\hline Niblinto & Pichimalleco & 800 & $38^{\circ} 10^{\prime} 13,38^{\prime \prime}$ & $71^{\circ} 49^{\prime} 15,84^{\prime \prime}$ & 200 \\
\hline Niblinto & Las Escuadras & 900 & $38^{\circ} 9^{\prime} 16,55^{\prime \prime}$ & $71^{\circ} 46^{\prime} 31,67^{\prime \prime}$ & 34 \\
\hline Niblinto & Los Mapochos & 1000 & $38^{\circ} 10^{\prime} 25,79^{\prime \prime}$ & $71^{\circ} 51^{\prime} 42,18^{\prime \prime}$ & 150 \\
\hline Niblinto & Macizo Sur Oeste & 1200 & $38^{\circ} 9^{\prime} 38,03^{\prime \prime}$ & $71^{\circ} 50^{\prime} 33,98^{\prime \prime}$ & 300 \\
\hline Niblinto & Las Quemas & 1350 & $38^{\circ} 9^{\prime} 57,88^{\prime \prime}$ & $71^{\circ} 48^{\prime} 25,57^{\prime \prime}$ & 80 \\
\hline
\end{tabular}

\section{AGRADECIMIENTOS}

A CONAF, a través de la Gerencia de Áreas Silvestres y al Departamento Áreas Silvestres Protegidas, Región de la Araucanía. En especial a Miguel Echeverría, administrador interino y a José Burgos, guardaparque encargado del Sector Prado 18, ambos de la Reserva Nacional Malleco, por su valiosa colaboración y apoyo en las actividades de terreno.

\section{BIBLIOGRAFÍA}

Benoit, I. 1989. Libro rojo de la flora terrestre de Chile (Primera parte). Corporación Nacional Forestal, Santiago. 157 pp.

Donoso, C. 1981. Tipos forestales de los bosques nativos. Documento de trabajo $\mathrm{N}^{\circ} 38$. Investigación y Desarrollo Forestal, CONAF-FAO. 70 pp.

Donoso, C. \& C. Ramírez. 1985. Arbustos nativos de Chile. Guía de reconocimiento. Colección Naturaleza de Chile, Vol. 2. Editorial Alborada, Valdivia.181 pp.

Hauenstein, E. \& M. SaAvedra. 2011. Plan de conservación de la Huella chica, Corynabutilon ochsenii (Phil.) Kearney, en Chile. CONAF. Santiago. 30 pp.

Hechenleitner, P., M.F. Gardner, P.I. Thomas, C. Echeverría, B. Escobar, P. Brownless \& C. Martínez. 2005. Plantas amenazadas del Centro-Sur de Chile: Distribución, Conservación y Propagación. Universidad Austral de Chile-Royal Botanic Garden Edinburgh, Valdivia, Chile. 188 pp.
Hoffmann, A. 1982. Flora Silvestre de Chile, Zona Araucana. Cuarta Edición, Ediciones Fundación Claudio Gay. Santiago 258 pp.

Hoffmann, A.1991. Flora Silvestre de Chile, Zona Austral. $2^{\mathrm{a}}$ ed. Ediciones Fundación Claudio Gay, Santiago. 258 pp.

Ministerio del Medio Ambiente. 2013. Ficha de antecedentes de la especie. ID 417. Noveno Proceso de Clasificación de Especies, Chile. URL: http://www.mma.gob.cl/ clasificacionespecies/fichas9proceso/Corynabutilon_ ochsenii.pdf.

Marticorena, A. 2005. Malvaceae. En: C. Marticorena \& R. Rodríguez (eds.), Flora de Chile, Vol. 2(3), pp. 22-105. Ediciones Universidad de Concepción, Chile.

Ross, C.A. 1987 Autoecología de Corynabutilon ochsenii Phil. (Malvaceae) en Chile. Tesis Ingeniero Forestal, Facultad de Ciencias Forestales, Universidad Austral, Valdivia, Chile. 64 pp.

Riedemann, P. \& G. Aldunate. 2003. Flora nativa de valor ornamental. Chile zona sur. Editorial Andrés Bello, Santiago. $516 \mathrm{pp}$

SaAvedra, M. 2007. Antecedentes técnicos de la huella chica (Corynabutilon ochsenii (Phil.) Kearney) en la Región de La Araucanía, para la elaboración de su plan nacional de conservación. CONAF Región de La Araucanía, Depto. de Patrimonio Silvestre. Temuco. 9 pp.

Serra, M.T., R. Gajardo \& A. Cabello. 1986. Fichas técnicas de especies amenazadas. Programa de protección y recuperación de la flora nativa de Chile. CONAF, Departamento de Áreas Silvestres ProtegidasUniversidad de Chile, Facultad de Ciencias Agrarias y Forestales, Departamento de Silvicultura y Manejo. Santiago. $17 \mathrm{pp}$.

Recibido: 16.06.14

Aceptado: 17.08.15 